\title{
Dextran induced acute kidney injury in a patient with dengue haemorrhagic fever
}

\author{
Priyacharana M P $\mathbf{P}^{1}$, Sumanadasa M H S1, Thiranagama P D K $\mathbf{K}^{2}$, Marasinghe $\mathbf{A}^{3}$, Shyamali $\mathbf{N}$ L $\mathbf{A}^{1,4}$ \\ Journal of the Ceylon College of Physicians, 2019, 50, 41-43
}

\begin{abstract}
Dextran 40 is a low molecular weight dextran preparation which is used as an adjunctive treatment in hypovolemic shock. At present dextran 40 is mainly used in the clinical practice for management of severe cases of dengue haemorrhagic fever or dengue shock syndrome. Acute kidney injury is a rare adverse reaction of dextran administration. We report a case of dextran induced acute kidney injury complicated with hypertensive encephalopathy during the convalescent phase of the illness. Renal biopsy revealed fine isometric vacuolations of tubular epithelial cells suggestive of osmotic nephrosis.
\end{abstract}

Key words: dextran induced acute kidney injury, dengue haemorrhagic fever, osmotic nephrosis

\section{Case presentation}

A 24-year-old man was admitted to the hospital on the 4th day of a febrile illness. Fever was high grade with associated arthralgia, myalgia, headache and vomiting. He had been in good health previously and denied a history of leptospirosis exposure.

On examination, he was febrile with temperature of $38.3^{\circ} \mathrm{C}$, pulse rate of $120 \mathrm{bpm}$ and supine blood

\footnotetext{
1 University Medical Unit, Colombo South Teaching Hospital, Sri Lanka.

${ }^{2}$ Histopathology Unit, Colombo South Teaching Hospital, Sri Lanka.

${ }^{3}$ Nephrology Unit, Colombo South Teaching Hospital, Sri Lanka.

${ }^{4}$ Department of Medicine, Faculty of Medical Sciences, University of Sri Jayewardenepura, Sri Lanka.
}

Corresponding author: Priyacharana M P

E-mail: pathmapriyacharana@gmail.com pressure of 110/80 $\mathrm{mmHg}$. Breath sounds were equal in both lungs and there was tenderness in the right hypochondrium. His body weight was $83 \mathrm{~kg}$. The haematochrit $(\mathrm{HCT})$ was $55 \%$ when measured using micro hematocrit method. Full blood count on ad-

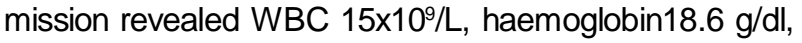
haematochrit (HCT) $51.8 \%$ and platelet count of $20 \times 10^{9} / \mathrm{L}$. Ultrasound scan of abdomen showed ascites with gall bladder wall oedema.

Diagnosis of dengue haemorrhagic fever (DHF) was made and he was started on intravenous fluid. During the first 24 hours he was given two $500 \mathrm{ml}$ boluses of $0.9 \%$ sodium chloride followed by intravenous $40 \%$ dextran $500 \mathrm{ml}$ due to persistently high haematocrit level, tachycardia and oliguria. Patient responded well to the dextran bolus with improvement of clinical parameters and urine output. However, on the 5th day of illness, he again required a normal saline bolus and $250 \mathrm{ml}$ of $40 \%$ dextran to stabilize clinical parameters. Secondary bacterial infection was suspected due to elevated neutrophil count and started on intravenous antibiotics.

Results of laboratory investigations during the hospital stay are shown below (Table 1,2). Twentyfour hours after the administration of dextran, his creatinine level was increased which continued to rise during next few days. Urine analysis showed albuminuria $(++)$, microscopic hematuria (10-12 red cells/ hpf) and 12 to 15 of pus cells/hpf without any casts. Ultrasound scan of abdomen showed normal sized kidneys with increased renal echogenicity suggestive of acute kidney injury (AKI). Serology was positive for dengue $\lg G$ \& $\lg M$ antibodies and leptospirosis antibody was negative on $7^{\text {th }}$ day of illness. His creatinine levels started to normalize after $8^{\text {th }}$ day of illness and urine output was satisfactory. On $13^{\text {th }}$ day of illness, he was discharged with the creatinine level of $399.4 \mu \mathrm{mol} / \mathrm{L}$ after conservative management

One week after the discharge, on $20^{\text {th }}$ day of illness, he presented to the emergency treatment unit with generalized tonic-clonic convulsions. His blood 
pressure was $196 / 130 \mathrm{mmHg}$ and there were no focal neurological signs or papilloedema. Non contrast CT scan of brain on admission was normal. Full blood count showed normal white cell count, haemoglobin of $11.1 \mathrm{~g} / \mathrm{dL}$ with platelet of $551 \times 10^{9} / \mathrm{L}$. Renal function tests revealed serum creatinine of $254.4 \mu \mathrm{mol} / \mathrm{L}$ with normal electrolyte levels including serum calcium and magnesium. Ultrasound scan of abdomen showed acute renal parenchymal changes and renal biopsy was carried out.

The light microscopic examination of the renal biopsy showed normal glomeruli. There were swelling and fine cytoplasmic vacuolations in the tubular epithelial cells. The vacuolations were isometric. The brush border of the tubular epithelial cells was preserved. Some tubules showed mild tubular dilatation. The interstitium showed mild acute on chronic inflammation with fibrosis and oedema (Figure 1). These tubular changes were compatible with changes seen in osmotic tubular injury.

He was managed as hypertensive encephalopathy secondary to AKI. He made an uneventful recovery and currently followed up at the nephrology clinic. There were no recurrent episodes of seizures and blood pressure was controlled with anti-hypertensive medications.

Table 1. Results of haematological investigations during the hospital stay

\begin{tabular}{l|l|l|l|l|l|l|l|l}
\hline \multirow{2}{*}{\multicolumn{1}{c|}{ Investigation }} & \multirow{2}{*}{$\begin{array}{l}\text { Normal } \\
\text { Range }\end{array}$} & \multicolumn{7}{|c}{ Days of illness } \\
\cline { 3 - 9 } & $4^{\text {th }}$ & $5^{\text {th }}$ & $6^{\text {th }}$ & $7^{\text {th }}$ & $8^{\text {th }}$ & $11^{\text {th }}$ & $13^{\text {th }}$ \\
\hline \multirow{2}{*}{ White blood cells (103/uL) } & $4.5-11$ & 15 & 24.29 & 24.74 & 21.11 & 17.69 & 11.68 & 9.04 \\
Neutrophils (\%) & $40-70$ & 74.2 & 63.6 & 56.4 & 64.5 & 73 & 90.8 & 91 \\
Lymphocytes (\%) & $22-44$ & 16.7 & 31.2 & 38.1 & 26.4 & 19.7 & 4.4 & 4.8 \\
Haemoglobin (g/dL) & $13.5-17.5$ & 18.6 & 14.7 & 13.7 & 12.5 & 12.6 & 10.9 & 10.5 \\
Haematocrit $(\%)$ & $41-53$ & 51.8 & 41.4 & 39.1 & 35.5 & 39.4 & 33.4 & 31.4 \\
Platelet count (10\%/L) & $150-400$ & 20 & 41 & 69 & 132 & 148 & 351 & 375 \\
\hline
\end{tabular}

Table 2. Results of biochemical investigations

\begin{tabular}{|c|c|c|c|c|c|c|c|}
\hline \multirow[t]{2}{*}{ Investigation } & \multirow{2}{*}{$\begin{array}{l}\text { Normal } \\
\text { Range }\end{array}$} & & & & & & \\
\hline & & $5^{\text {th }}$ & $6^{\text {th }}$ & $7^{\text {th }}$ & $8^{\text {th }}$ & $11^{\text {th }}$ & $13^{\text {th }}$ \\
\hline Aspartate aminotransferase $(\mathrm{u} / \mathrm{L})$ & $10-40$ & 3279.4 & 2291.5 & 1756.9 & 781.1 & & 109.4 \\
\hline Alanine aminotransferase $(\mathrm{u} / \mathrm{L})$ & $10-55$ & 1391.3 & 1198.5 & 1131.4 & 682.9 & & 245.8 \\
\hline Total Bilirubin $(\mu \mathrm{mol} / \mathrm{l})$ & $5-21$ & 16.5 & & & 23.4 & & 10.19 \\
\hline Serum Albumin (g/l) & $35-52$ & 24.3 & & & 29.3 & & \\
\hline Sodium (mmol/l) & $136-146$ & 132.6 & 133 & 135 & 135.5 & 130.2 & 130.4 \\
\hline Potassium (mmol/l) & $3.5-5.1$ & 5.2 & 4.8 & 4.3 & 3.9 & 4.1 & 4.2 \\
\hline Serum Creatinine $(\mu \mathrm{mol} / \mathrm{l})$ & $74-110$ & 278.6 & 420.7 & 454.7 & 607 & 439.5 & 399.4 \\
\hline Blood urea $(\mathrm{mmol} / \mathrm{l})$ & $2.8-7.2$ & 18.2 & 26.8 & 25.2 & 28.8 & 38.7 & 36.7 \\
\hline
\end{tabular}




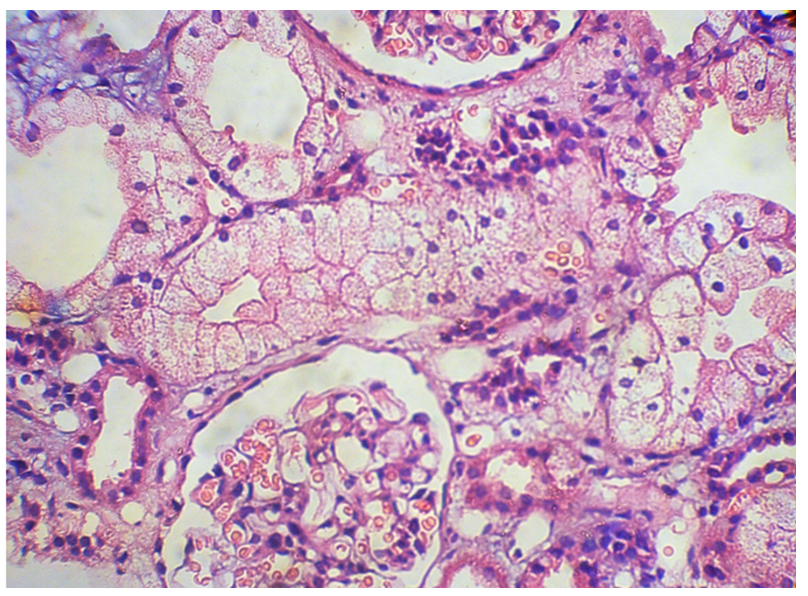

Figure 1. Fine vacuolations of tubular epithelial cells.

\section{Discussion}

Dengue is the most prevalent mosquito borne viral infection in Sri Lanka. While the majority of individuals who are infected with the dengue virus develop asymptomatic or an undifferentiated viral fever like illness, it can cause severe clinical manifestations such as dengue hemorrhagic fever leading to shock and organ involvement in $10-25 \%$ of individuals ${ }^{1}$.

Renal involvement of dengue is uncommon and can manifest in several forms including proteinuria, hematuria, AKI, acute tubular necrosis, hemolytic uremic syndrome and glomerulonephritis ${ }^{2}$. Development of $\mathrm{AKI}$ in dengue infection carries a poor prognosis and associated with increased mortality ${ }^{3}$. Patients who develop AKI prior to hospital admission carry a higher risk of mortality compared to the patients who develop AKI during hospital stay4.

DHF is characterized by plasma leakage and abnormal hemostasis due to increased capillary permeability and endothelial cell injury ${ }^{5,6}$. Plasma leakage is transient and usually lasts for 24 to 48 hours ${ }^{6}$. Dengue shock syndrome (DSS) is hypovolemic shock caused by plasma leakage and patients with severe DHF and DSS may require intravenous fluid therapy with colloidal solutions in addition to conventional crystalloid solutions ${ }^{5}$. Most commonly used effective colloidal solution is dextran 40 which is low molecular weight dextran, a mixture of glucose polymers of various sizes ${ }^{7}$. Dextran 40 is excreted in urine by rapid filtration through glomeruli and affect urine osmolality ${ }^{5,7}$. However, it may circulate in the system up to 24 hours $^{7}$. Due to the renal effects, the dose of dextran is restricted to $30 \mathrm{ml} / \mathrm{kg} /$ day in the management of $\mathrm{DHF}^{5}$.

Each gram of dextran increases plasma oncotic pressure by 6-7 Hg mm. Reduction of filtration pressure and glomerular filtration rate (GFR) due to this elevated plasma oncotic pressure is thought to be the mechanism of dextran induced $\mathrm{AKI}^{7}$. Afferent arteriole vasoconstriction caused by increased urinary solute excretion could be contributing to reduction of GFR ${ }^{7}$. Advanced age, renal artery stenosis, cardiovascular diseases, dehydration and previous renal insufficiency predispose to the development of dextran induced $\mathrm{AKI}^{7}$. Histological assessment of kidneys affected by dextran induced AKI has revealed, osmotic nephrosis without any evidence of glomerular pathology or tubular necrosis ${ }^{7}$.

Osmotic nephrosis is characterized by the morphological pattern with vacuolization and swelling of proximal tubular cells and diagnosed only by renal biopsy $^{8}$. It is a reversible, functional form of renal injury which recovers after the discontinuation of the causative agent $^{7,8}$. Plasmapheresis will be required for removal of dextran molecules from circulation, as removal by hemodialysis will be ineffective due to its molecular weight ${ }^{7,8}$.

Renal biopsy of our patient showed characteristic appearance of osmotic nephrosis, which confirmed the diagnosis of dextran-induced AKI. Though rare, as dextran can induce $A K I$, it should be reserved for patients who really need it.

\section{References}

1. Fernando S, Wijewickrama A, Gomes L, Punchihewa CT, et al. Patterns and causes of liver involvement in acute dengue infection. BMC Infectious Diseases. 2016; 16(1): 319.

2. Oliveira JF, Burdmann EA. Dengue-associated acute kidney injury. Clinical Kidney Journal 2015; 8(6): 681-5.

3. Lizarraga KJ, Nayer A. Dengue-associated kidney disease. Journal of Nephropathology 2014; 3(2): 57-62.

4. Mallhi TH, Khan AH, Adnan AS, Sarriff A, Khan YH, Jummaat F. Incidence, Characteristics and Risk Factors of Acute Kidney Injury among Dengue Patients: A Retrospective Analysis. PLoS One 2015; 10(9): e0138465.

5. WHO. Comprehensive guideline for prevention and control of dengue and dengue haemorrhagic fever. $2011 \mathrm{http}: / /$ www.searo.who.int/entity/vector_borne_tropical_ diseases/documents/SEAROTPS60/en/.

6. Kularatne SA, Weerakoon KG, Munasinghe R, Ralapanawa UK, Pathirage M. Trends of fluid requirement in dengue fever and dengue haemorrhagic fever: a single centre experience in Sri Lanka. BMC Research Nnotes 2015; 8: 130.

7. Ferraboli R, Malheiro PS, Abdulkader RCRM, Yu L, Sabbaga E, Burdmann EA. Anuric Acute Renal Failure Caused by Dextran 40 Administration. Renal Failure 2009; 19(2): 303-6.

8. Dickenmann M, Oettl T, Mihatsch MJ. Osmotic nephrosis: acute kidney injury with accumulation of proximal tubular lysosomes due to administration of exogenous solutes. American Journal of Kidney Diseases 2008; 51(3): 491-503. 